\title{
Betriebliches Gesundheitsmanagement Mitarbeiter und Klinik profitieren
}

Die Schön Klinik setzt auf Zufriedenheit, Gesundheit und Vorsorge für die eigenen Mitarbeiter. Denn nur wer gesund sei, könne sich optimal um seine Patienten kümmern, sagte Geschäftsführer Dr. Michael Knapp anlässlich der Verleihung des Corporate Health Awards 2015 in Bonn. Die inhabergeführte Klinikkette mit Hauptsitz in Prien am Chiemsee mit 17 Standorten und 9000 Beschäftigten gewann den Preis für ein vorbildliches Betriebliches Gesundheitsmanagement (BGM) zum zweiten Mal in Folge. Sie behauptet sich damit unter $\mathbf{3 0}$ Mitbewerbern in der Kategorie Gesundheit und Soziales. Drei Häuser zählen zudem zu den 10 besten Häusern nach den Kriterien von Great Place to Work 2014. Gesundheitsmanagerin Michaela Preis kümmert sich darum, dass die strategische Ausrichtung in puncto Mitarbeitergesundheit tatsächlich umgesetzt wird.

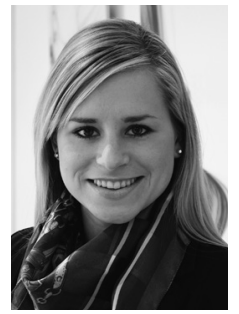

Michaela Preis
Vor welcher Herausforderung standen Sie, als Sie vor 3 Jahren die Verantwortung für das Betriebliche Gesundheitsmanagement übernommen haben, Frau Preis?

Michaela Preis: Ein BGM mit den klassischen Maßnahmen zur Gesundheitsförderung wird seit 2006 in den Kliniken angeboten. Dabei spielten und spielen heute unsere BGM-Teams in den Kliniken eine sehr wichtige Rolle. Sie organisieren Aktionen und Maßnahmen von Mitarbeitern für Mitarbeiter. Zum Beispiel bieten Therapeuten Yoga-Kurse an. Als ich in Prien anfing, ging es darum, die Mitarbeitergesundheit und -zufriedenheit in den Vordergrund zu rücken und ein strukturiertes BGM-Konzept zu erarbeiten, das einheitliche Strukturen für die 17 Klinikstandorte vorgibt, aber dennoch genügend Flexibilität bietet, um auf die jeweiligen Bedürfnisse und Herausforderungen der Standorte einzugehen.

\section{? Was zeichnet das BGM der Schön} Kliniken heute aus?

Michaela Preis: Das Besondere ist das große Engagement, mit dem die Kliniken an allen Standorten das BGM vertreten. Anders wären die Erfolge sicherlich nicht machbar. In jeder Klinik gibt es einen Arbeitskreis Gesundheit und ein BGMTeam für die konkreten Maßnahmen. Der Arbeitskreis trifft sich einmal pro Quartal. $\mathrm{Zu}$ unseren Vorgaben gehört, dass die Personalleitung, der Betriebsrat, die Arbeitssicherheit, der Betriebsarzt und ein BGMKoordinator vertreten sein müssen. Die Kliniken dürfen natürlich weitere Personen hinzuziehen, zum Beispiel die Schwerbehindertenvertretung, die Pflegedienstleistung und den Sozialdienst. Aus Sicht der Zentrale ist wichtig, dass die Ausrichtung stimmt und bestimmte Verfahren eingehalten werden. Dazu zählen unter anderem die regelmäßigen Treffen der Arbeitskreise und die Evaluation jeder BGM-Maßnahme.

Welchen Input liefert die Beteiligung am Corporate Health Award? Michaela Preis: Die Audits waren sehr hilfreich, schon in den Vorjahren, bevor wir den Award erhielten. Sie schaffen einen Branchenvergleich und zeigen auf, wo Entwicklungsmöglichkeiten bestehen. Die externen Experten betonten, wie wichtig es sei, immer wieder Feedback bei den Mitarbeitern einzuholen, um festzustellen, was eine BGM-Maßnahme bringt. Sie empfahlen, zum Beispiel nach kurzfristigen und langfristigen Kennziffern zu unterscheiden, so lässt sich die Teilnahme an den Maßnahmen schnell ermitteln. Anders sieht es bei den langfristigen Effekten aus, die wir uns für die Gesundheit der Mitarbeiter versprechen. Der Krankenstand ist sicherlich ein wichtiger Indikator, aber alleine nicht aussagekräftig genug.

\section{? Wie nehmen Mitarbeiter die}

\section{BGM-Maßnahmen wahr?}

Michaela Preis: Zu den Angeboten erhalten wir durchweg positive Rückmeldung. Vor allem die Sportkurse werden sehr gut angenommen. Schwierig ist es eher, die Angebote mit den Arbeitsabläufen zu vereinbaren, so findet zum Beispiel der SportKurs direkt nach den Schichten statt oder die Aktive-Pause in den Pausenzeiten der Mitarbeiter. Außerdem ist es nicht immer einfach, Mitarbeiter für die eigene $\mathrm{Ge}$ sundheit zu sensibilisieren. Führungskräfte können dies jedoch beeinflussen, indem sie ihre eigene Gesundheit und die ihrer Mitarbeiter im Blick haben. Wir bieten daher Schulungen zum Thema „Gesund führen“ an. Ebenso ist das Thema Gesundheit in unserem FührungskräftenachwuchsProgramm fest verankert, um auch unsere jungen Führungskräfte von Beginn an für das Thema Gesundheit zu sensibilisieren.

Lassen sich Unterschiede zwischen den Standorten erkennen?

Michaela Preis: Ja, definitiv. Die Unterschiede ergeben sich schon durch die unterschiedliche Fachrichtung. Eine Klinik für Orthopädie setzt eher auf körperlich bezogene BGM-Maßnahmen, während in der Neurologie psychische Faktoren stärker Beachtung erfahren. Die Maßnahmen müssen daher sehr klinikindividuell zugeschnitten sein und zu den Arbeitsabläufen und Mitarbeitern passen. Im Kern geht es darum, am Ball zu bleiben, und die Aktionen an den Bedarfen vor Ort auszurichten.

\section{Stellen Ärzte eine besondere}

\section{Zielgruppe dar?}

Michaela Preis: Nein, das kann man so nicht sagen, vielmehr hat jede Berufsgruppe ihre eigenen Herausforderungen, auf die wir mit den BGM-Angeboten eingehen müssen. Die verschiedenen Berufsgruppen müssen miteinander konstruktiv arbeiten können. Als besondere Zielgruppe sind eher die Führungskräfte zu nennen, dies ist jedoch losgelöst von der Berufsgruppenzugehörigkeit. Ziel ist, alle Beschäftigten gesund zu erhalten.

Rechnet sich ein BGM betriebswirtschaftlich? Sollte es sich rechnen?

Michaela Preis: Ich bin überzeugt, dass sich ein BGM rechnet, und es muss sich rechnen. Letztendlich sollten sowohl die Mitarbeiter als auch das Unternehmen davon profitieren. Ein Return on Invest lässt sich langfristig nachweisen - das haben bereits mehrere Unternehmen bewiesen. Wir ermitteln dies anhand eines Kennzahlen-Mix. Hierzu gehören Kennzahlen zur Teilnehmerzufriedenheit mit den BGM-Maßnahmen, die Ergebnisse der Mitarbeiterumfrage sowie Daten über Arbeitsausfallzeiten und Arbeitsunfälle.

Frau Preis, vielen Dank für das Gespräch!

Das Interview führte Dr. Adelheid Weßling, freie Journalistin, Düsseldorf. 